therefore biochemistry laboratories throughout UK receive a substantial number of requests for coeliac serological assessments every year.

Objective We set out to review the total number of requested anti-endomysial antibody (AEAs) and anti-tissue transglutaminase (ATTG) tests between 2007 and 2013 at a busy district general hospital, and reviewed the outcome of having made that request. Methods The results of all the coeliac serology requests made during the study period were reviewed and a retrospective analysis was made of the hospital records to find out the outcome in all those patients with positive results. The laboratory issued a positive result if the ATTG was greater or equal to 4 .

Results During 2007, 810 AEAs were requested, of which 30 $(3.7 \%)$ were positive (10 were weak positive), 736 were negative, 44 were not done. In 2008 ATTG became the primary coeliac serology test and AEAs were only used to review gluten free dietary (GFD) compliance. Between Jan 2008 and Dec 2013 a total of 20,677 ATTGs were requested. This has steadily increase year on year. In 2008 there were 913 requests, 1,389 in $2009,3,060$ in 2010, 4,238 in 2011, 5,584 in 2012 and 6,483 in 2013 . Of these 785 (3.6\%) proved positive, 19891 were negative, 372 samples were deemed insufficient and 605 were rejected by the laboratory as not indicated. Histological confirmation of coeliac disease was made in 222 patients, however a large proportion of positive serology received no further assessment.

Conclusion There is an ever increasing number of requests for coeliac serology, costing our local CCG $£ 21,070$ in 2013. Despite the positive pick up rate being high at $3.6 \%$, a large number of positive results were not pursued any further, with patients failing to have a definitive diagnosis made. It is important to ensure that there are robust mechanisms of chasing up on hospital results, and acting on them appropriately to prevent delayed or missed diagnoses.

Disclosure of Interest None Declared.

\section{PTU-161 ENDOSCOPIC BOUGIE DILATATION IS EFFECTIVE AND SAFE FOR OES OPHAGEAL AND PHARYNGEAL STRICTURES: OUTCOMES OF A LARGE CASE SERIES}

MR Smith*, B Drinkwater, M Widlak, NC Fisher. Gastroenterology, Dudley Group Hospitals NHS Foundation Trust, Dudley, UK

\subsection{6/gutjnl-2014-307263.235}

Introduction Endoscopic bougie dilatation is a traditional technique for managing oesophageal strictures. There are some safety concerns with this technique, but no corroborative evidence of this in controlled or uncontrolled studies to date.

Methods We evaluated the outcomes and safety of endoscopic bougie dilatation at our centre, using the endoscopy database to identify all dilatations done by a single operator. Bougies were the preferred option for all dilatations. All cases from January 2007 to March 2013 were then reviewed by case note analysis.

Results 146 patients were identified, who underwent a total of 346 bougie dilatations. Median age was 67 yrs (range 27-91). Indications for dilatation were: peptic stricture 80 (55\%), malignant stricture $20(14 \%)$, post-surgical stricture $12(8 \%)$, pharyngeal pathology $25(17 \%)$ and other 9 (6\%). Pharyngeal pathology was predominantly post-radiotherapy strictures (64\%) and neurological (20\%).

In cases of peptic stricture, $78 / 80(98 \%)$ had a good symptomatic response to an initial course of dilatation (requiring 1 procedure only in $82 \%$ ). Median end dilatation diameter was
$17 \mathrm{~mm}$ (range 12-18). Recurrence requiring further dilatation occurred in 27 (34\%), after a median of 8 months (range 3-47). In the remainder, median observed remission was 24 months (range 1-63). For pharyngeal pathology patients underwent a median of 2 dilatations (range 1-12). After initial dilatation, 12 (48\%) achieved lasting benefit, 5 (20\%) had no benefit and 8 (32\%) benefited from periodic scheduled dilatations.

Overall median follow up was 22 months (IQR 7- 48). Among the whole case series there were $6(4 \%)$ unscheduled admissions, all self-limiting (dysphagia 2, food bolus 2, stentrelated bleed 1, pain 1). There were no perforations.

Conclusion This large case series supports the role of bougie dilatation as a safe and effective therapy for benign peptic strictures. With careful case selection it also appears a valuable, appropriate and safe option for a range of similar oesophageal and pharyngeal pathologies.

Disclosure of Interest None Declared.

\section{PTU-162 THE EPIDEMIOLOGY, CLINICOPATHOLOGICAL CHARACTERISTICS ND OUTCOMES OF GISTS IN THE NORTH EAST OF ENGLAND OVER A FIVE YEAR PERIOD}

${ }^{1}$ MJ Friel* ${ }^{*}{ }^{2} Y$ Vishwanath, ${ }^{2} \mathrm{H}$ Wescott, ${ }^{3} \mathrm{~N}$ Wadd, ${ }^{1} \mathrm{~A}$ Dhar. ${ }^{1}$ Gastroenterology, County Durham and Darlington NHS Foundation Trust, Co. Durham, UK; ${ }^{2}$ Surgery, James Cook University Hospital, Middlesbrough, UK; ${ }^{3}$ Oncology, James Cook University Hospital, Middlesbrough, UK

\subsection{6/gutjnl-2014-307263.236}

Introduction Gastrointestinal stromal tumours (GISTs) are rare mesenchymal tumours of the gastrointestinal tract. In recent years there is increasing focus on immunohistochemistry biomarkers and targeted Imatinib therapy for treatment, but there is little data from the UK on factors that influence outcome.

Methods We reviewed clinical, pathological, treatment strategies, follow-up and outcome data in all patients with GISTs at our regional multidisciplinary cancer group between Jan 2008 and Dec 2012. Tumour size, mitotic index, other pathological parameters and immunohistochemical stains including CD117 (KIT), CD34, and others were recorded. Tumours were categorised according to the NIH, revised AFIP, and AJCC risk-stratification models. Cox proportional hazard regression was used to determine independent factors associated with survival.

Results 42 patients with GIST were identified. 36(85.7\%) were located in the stomach, $5(11.9 \%)$ in the small intestine, and 1 $(2.4 \%)$ in the oesophagus. Median age was 68 (range 43-91) yrs. $24(57.1 \%)$ were female. Tumour size ranged from 1.0$12.7 \mathrm{~cm}$ (mean $5.5 \mathrm{~cm}$ ). Metastasis was present in $19(45.2 \%)$ at diagnosis, the liver being the most common site in $8(42.1 \%)$. Histology and immunohistochemical analysis was available in 31 (73.8\%). Commonest histological subtype was spindle cell in 17 (53.1\%), epitheloid in 9 (29.0\%) and mixed in 5 (16.1\%). CD117 was positive in 90.6\%, and CD34 in 75.0\%. 54.8\% patients underwent surgical resection with radical surgery in $47.8 \%, 5$ of whom received extensive adjacent organ resection. $47.8 \%, 34.8 \%$ and $52.2 \%$ patients were categorised as high risk according to NIH, AFIP and AJCC (stage III-IV) risk models respectively. Recurrence was confirmed in $5(11.9 \%)$ patients at a median of 597 (range 402-943) days. Of these, 2 were deemed low risk by all three classification systems. Imatinib was given to $14 / 42(33.3 \%)$ patients; as primary therapy in $10(28.3 \%)$ patients (9 palliative and 1 neoadjuvant), and as adjuvant therapy in 4 patients. Cox proportional hazard regression revealed age, tumour site, size, mitotic count, metastases at 
diagnosis and AFIP scores to be independently associated with worse prognosis.

Conclusion A combination of surgery and imatinib may be necessary to provide a curative treatment for GISTs and prevent recurrence. Although our study is limited by small numbers, current risk-categorisation models appear to over-estimate recurrence risk with discrepancies in predicting behaviour for certain low-risk tumours. A weighted scoring system combining independent factors associated with poor prognosis may serve as a more accurate clinical prediction tool.

Disclosure of Interest M. Friel: None Declared, Y. Vishwanath: None Declared, H. Wescott: None Declared, N. Wadd: None Declared, A. Dhar Consultant for: Honoraria for advisory board to the Pharmaceutical Industry, Speaker bureau with: Falk Pharma UK, Warner Chilcott UK, Shire Pharmaceuticals, Ferring Pharmaceuticals.

\section{PTU-163 URINARY VOLATILE ORGANIC COMPOUND ANALYSIS TO DISTINGUISH COELIAC DISEASE FROM IRRITABLE BOWEL SYNDROME: A PILOT STUDY}

IJ Covington, ${ }^{2} \mathrm{M}$ McFarlane*, ${ }^{3} \mathrm{R}$ Harbord, 'E Westenbrink, ${ }^{2} \mathrm{~S}$ Chambers, ${ }^{2} \mathrm{~A}$ Dhaliwal, ${ }^{2} \mathrm{~N}$ O'Connell, ${ }^{2} \mathrm{C}$ Bailey, ${ }^{2} \mathrm{C}$ Nwokolo, ${ }^{4} \mathrm{~K}$ Bardhan, ${ }^{2,5} \mathrm{R}$ Arasaradnam. ${ }^{1}$ School of Engineering, University of Warwick, Coventry, UK; ${ }^{2}$ Gastroenterology, UHCW, University of Warwick, Coventry, UK; ${ }^{3}$ MOAC Doctoral Training Centre, University of Warwick, Coventry, UK; ${ }^{4}$ Gastroenterology, Rotherham General Hospital, Rotherham, UK; ${ }^{5} \mathrm{CSRI}$, University of Warwick, Coventry, UK

\subsection{6/gutjnl-2014-307263.237}

Introduction Coeliac disease (CD), a T-cell-mediated gluten sensitive enteropathy, affects $\sim 1 \%$ of the UK population, and in adults presents with a wide range of clinical features; often mistaken for irritable bowel syndrome (IBS). Heightened clinical awareness and serological screening identifies those likely to have $\mathrm{CD}$; the diagnosis confirmed by histological features in small bowel/duodenal biopsies. Limitations to diagnosis are false negative serology (e.g., in IgA deficient patients, the young and the elderly) and reluctance to undergo biopsy. Examining the pattern of urinary volatiles offers a novel non-invasive approach. The gut microbiome is perturbed in several gastrointestinal disorders, resulting in altered gut fermentation patterns, and recognisable by analysis of volatile organic compounds (VOC) in urine, breath and faeces. The altered structure of the small intestinal mucosa, increased gut permeability and altered gluten peptide metabolism, we hypothesised, would change the

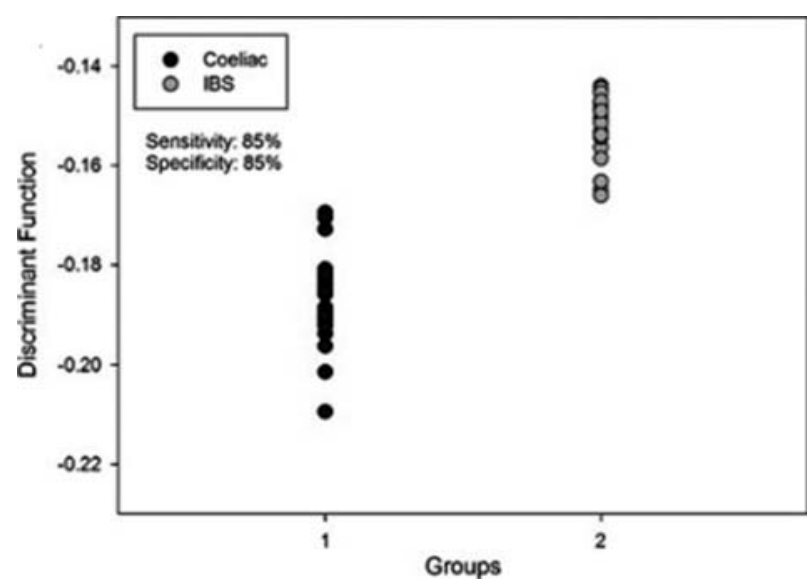

Abstract PTU-163 Figure 1 microbiome creating a unique "fermentome" pattern, distinguishable from IBS. We investigated this by examining the urinary VOC pattern using Field Asymmetric Ion Mobility Spectrometry (FAIMS).

Methods 47 patients were recruited, 27 with $\mathrm{CD}$ and 20 with diarrhoea-predominant IBS (D-IBS). Urine was collected and $10 \mathrm{ml}$ aliquots were stored frozen in universal containers. For assay, the containers were heated to $40 \pm 0.1^{\circ} \mathrm{C}$. The headspace above the sample was then analysed by FAIMS. Linear discriminant analysis (LDA) was used for statistical evaluation.

Results LDA showed that FAIMS distinguishes the VOC pattern in CD vs D-IBS with a sensitivity and specificity of $85 \%$ respectively.

Conclusion This pilot study suggests that FAIMS offers a novel non-invasive approach to identify those likely to have $\mathrm{CD}$, and distinguishes from D-IBS. It may have the potential to non-invasively track the progress of $\mathrm{CD}$ when on a gluten-free diet, to monitor adherence and observe changes.

Disclosure of Interest None Declared.

\section{PTU-164 EVIDENCE OF TWO AETIOLOGIES OF GASTROESOPHAGEAL JUNCTIONAL CANCERS BASED ON GASTRIC PARIETAL CELL DENSITY}

${ }^{1} \mathrm{MH}$ Derakhshan*, ${ }^{2} \mathrm{~T}$ Harvey, ${ }^{2} \mathrm{R}$ Ferrier, ${ }^{1} \mathrm{EV}$ Robertson, ${ }^{2} \mathrm{C}$ Orange, ${ }^{2} \mathrm{M}$ Forshaw, ${ }^{2} \mathrm{JJ}$ Going, ${ }^{1} \mathrm{KE}$ McColl. ${ }^{1}$ Institute of Cardiovascular and Medical Sciences, University of Glasgow, Glasgow, UK; ${ }^{2}$ Institute of Cancer Sciences, University of Glasgow, Glasgow, UK

\subsection{6/gutjnl-2014-307263.238}

Introduction Serum pepsinogen I:II ratio, a surrogate marker of atrophic gastritis, suggests that some adenocarcinomas at the gastroesophageal junction (GOJ) develop on a background of atrophic gastritis, similar to non-cardia gastric cancer, while others arise on a backgrounds of healthy, non-atrophic gastric mucosa similar to oesophageal adenocarcinoma. In this current study, we have directly the background gastric body mucosa in patients with junctional adenocarcinomas compared to oesophageal adenocarcinomas and non-cardia gastric cancers.

Methods 127 gastrectomy and oesophagectomy specimens for adenocarcinoma were identified for which clear topographic description allowed assignment to oesophageal, junctional (including cardia) and gastric non-cardia locations. In these gastric body mucosa specimens, well clear of the tumour margin, parietal cells were immunostained using anti- $\mathrm{H}^{+} / \mathrm{K}^{+}$ATPase. Parietal cell density was counted in 3 to 5 well-oriented fields $\left(1 \mathrm{~mm}^{2}\right.$ each) and expressed as mean parietal cell number per $1 \mathrm{~mm}^{2}$ area. Total mucosal thickness, glandular thickness, intestinal metaplasia, inflammation indicated by polymorphonuclear (PMN) and mononuclear (MN) cells and reactive atypia (RA) were also scored. Non-parametric statistics were used to compare distributions.

Results Ten (8\%) cases lacked well-orientated blocks of body mucosa. The remaining 117 patients included 34 oesophageal, 52 GOJ and 31 non-cardia gastric adenocarcinomas. Median (IQR) parietal cell densities were 836 (173), 602 (389) and 411 (334) per $\mathrm{mm}^{2}$ in gastric mucosa of oesophageal, GOJ and gastric cancers, respectively (all differences $\mathrm{P}<0.001$ ). Using a parietal cell density of $630 / \mathrm{mm}^{2}, 85 \%$ of oesophageal adenocarcinomas had a higher and $84 \%$ of non-cardia gastric cancers had a lower values. With the same cut-off, 50\% of GOJ adenocarcinomas were gastric and remaining was oesophageal in origin.

Glandular mucosa was thicker in patients with GOJ cancer compared to gastric $(0.735$ vs. $0.600, p=0.005)$ and thinner 